\title{
Cartographie semi-automatisée et classification des réseaux de tranchées et boyaux du champ de bataille de Verdun à partir du LiDAR aéroporté
}

\author{
Semi-automated mapping and classification of trench networks and \\ casings on the Verdun battlefield using Airborne LiDAR
}

\author{
Rémi De Matos-Machado ${ }^{1}$, Jean-Pierre Toumazet ${ }^{2}$, Stéphanie Jacquemot ${ }^{3}$ \\ ${ }^{1}$ université de Lorraine, EA 7304 LOTERR, attaché temporaire d'enseignement et de recherche, 57000 Metz, France \\ ${ }^{2}$ université Clermont-Auvergne, CNRS, GEOLAB, maître de conférences, F-63000 Clermont-Ferrand, France \\ ${ }^{3}$ DRAC Grand Est, Service Régional de l'Archéologie de Metz, ingénieur d'études, 57000 Metz, France
}

RÉSUMÉ. En 2013, une mission LiDAR aéroportée menée sur le champ de bataille de Verdun a permis de mettre en évidence les reliefs induits par la Grande Guerre. Dissimulés par un vaste couvert forestier de 10000 ha, ces reliefs appelés polémoformes, en référence aux travaux de J.-P. Amat $(1987,2001,2015)$, appartiennent au patrimoine de la dramatique bataille de 1916 et constituent une réserve archéologique de premier ordre pour les générations futures. Malgré leur densité, les vestiges doivent être inventoriés de manière exhaustive dans le cadre du label national " forêt d'exception du champ de bataille de Verdun ". Cependant, à l'échelle du massif tout entier, ce travail n'est envisageable que si une méthode de cartographie automatisée est développée. Cet article détaille la démarche méthodologique mise en œuvre, à l'échelle des réseaux de tranchées et boyaux uniquement. La méthodologie utilisée est triple : (1) Elle consiste à extraire les formes du modèle numérique de terrain au moyen d'un algorithme semi-automatique ; (2) Le tracé des formes est étudié à l'aide d"un indice de sinuosité ; (3) L'interprétation des cartes d'inventaire réalisées est effectuée sur le terrain et complétée par des documents historiques récoltés dans des centres d'archives. La carte obtenue révèle près de $420 \mathrm{~km}$ de tranchées et boyaux. Leurs formes variées sont dues aux différentes manières d'aménager le front. Alors qu'une grande partie du réseau initial a disparu au cours du siècle dernier par érosion, le croisement des données LiDAR avec les documents d'archives permet d'estimer l'importance de cette perte et de localiser les secteurs affectés. Au-delà de la nature reproductible de la méthode, susceptible de faciliter la recherche sur d'autres champs de bataille, cette approche fournit des outils opérationnels pour la gestion et la conservation du patrimoine culturel de la forêt de Verdun. De ce fait, les corpus iconographiques et cartographiques produits seront directement utilisés dans le prochain plan d'aménagement forestier, afin d'optimiser la sauvegarde des polémoformes et des vestiges associés.

ABSTRACT. In 2013, an airborne LiDAR mission conducted over the Verdun battlefield has brought to light World War IOne landforms. Concealed by a large forest cover of 10,000 ha, these reliefs, called polemoforms in reference to J.-P. Amat's research $(1987,2001,2015)$, are direct remnants from the 1916 battle and constitute a prime archeological reserve for the future generations. Despite the high density of features, they should be exhaustively inventoried in the framework of the national label "forêt d'exception du champ de bataille de Verdun". However, at the scale of the entire site, this work is only feasible if an automated mapping method is developed. This paper especially focuses on the trench network inventory. The methodology used is threefold: (i) Landforms are extracted from the digital terrain model by means of a semi-automatic algorithm; (ii) Trench geometry is studied using a sinuosity index; (iii) Map interpretation is carried out on the field site and supplemented by historical documents collected in French and German archives centers. The resulting maps reveal approximately $420 \mathrm{~km}$ of fire and communication trenches. Their morphological signature is rich and due to the different ways of building facilities. Although a large part of the initial network disappeared during the last century, the crossing of LiDAR data with historical archives allows to estimate the length of the erased features and to locate the affected areas. Beyond the reproducible nature of the method, which may contribute to the research development on other battlefields, this research provides operational tools for management and conservation of the historical, cultural and natural heritage of the Verdun forest. As a result, the produced iconographic and cartographic corpus will be directly used in the next forest management plan, in order to optimize the safeguarding of polemoforms and associated remnants.

MOTS-CLÉS. LiDAR, polémoforme, forêt de Verdun, inventaire de formes, classification morphométrique.

KEYWORDS. LiDAR, polemoform, forest of Verdun, landform inventory, morphometric classification, automatic detection. 


\section{Introduction}

Avec l'essor des technologies digitales et dans le contexte des commémorations du centenaire de la Première Guerre mondiale, les recherches sur les polémoformes, formes de relief induites par les conflits passés (Amat, 1987, 2001, 2015), se sont multipliées. Cette volonté de connaître avec précision le nombre et l'état de ces traces, en particulier en contexte forestier où elles sont le mieux conservées, est étroitement liée à leur haute valeur patrimoniale. De fait, les polémoformes sont l'un des derniers témoins physiques des guerres passées. Dans le cas de la Première Guerre mondiale, ces reliefs constituent avec les documents d'archives les seuls éléments matériels permettant d'entretenir un lien direct avec le passé. L'étude des polémoformes forme aussi une manière d'appréhender les paysages de guerre en tant qu'objet archéologique. Les réseaux de fossés (tranchées, boyaux), les restes d'abris, les batteries d'artillerie, les cratères d'obus représentent autant de vestiges permettant de comprendre la vie des soldats sur le front et leur appropriation du milieu (Jacquemot et Legendre, 2011 ; Schnitzler et Landolt, 2013 ; Georges-Leroy et al., 2014 ; Jacquemot, 2014 ; Landolt, 2018). Ces vastes paysages anthropisés sont les témoins d'un passé douloureux qui conservent quantité d'objets de la vie quotidienne, mais également des dépouilles de soldats, comme en témoigne le nombre des restes humains mis au jour régulièrement lors de travaux. C'est dans ce contexte que de nombreuses recherches sur le patrimoine culturel des forêts de guerre ont vu le jour pendant les quatre années de commémoration (Devos et al., 2015; De Matos-Machado et al., 2016; Magnini et al., 2016; Stichelbaut et Cowley, 2016; Stichelbaut et al., 2016; Brenot et al., 2017 ; Stichelbaut et al., 2017 ; Taborelli et al., 2017a et 2017b; Gheyle et al., 2018 ; Note et al., 2018). Bien que prometteuses, les études susmentionnées ont souvent été confrontées au problème récurrent de la cartographie d'un nombre conséquent d'objets (i.e. digitalisation manuelle). Il fallait donc rechercher des méthodes d'inventaire automatisées capables de traiter le million de reliefs présent sur le champ de bataille. À l'heure actuelle, seulement deux études ont tenté de cartographier par voie automatique des polémoformes, en France et en Italie (Brenot et al., 2017 ; Magnini et al., 2016). Par ailleurs, rares sont les travaux discutant de la variabilité morphométrique des polémoformes et proposant des classifications de formes pouvant servir de modèle sur des sites peu connus. Pour pallier ces insuffisances, cet article propose une méthode de cartographie semi-automatique alternative, appliquée aux $100 \mathrm{~km}^{2}$ du champ de bataille de Verdun (rive droite). Il complète notre précédente publication (De Matos-Machado et al., 2019) en s'intéressant cette fois-ci à l'inventaire des réseaux de fossés.

\section{Site d'étude}

Le champ de bataille de Verdun constitue aujourd'hui une vaste région forestière (9615 ha) localisée à environ $200 \mathrm{~km}$ à l'est de Paris dans le département de la Meuse. La zone étudiée se situe au nord-est de Verdun et en rive droite de la Meuse, en forêt domaniale de Verdun, absente de ce territoire à la veille de la Première Guerre mondiale (fig. 1). Sa partie occidentale occupe le plateau calcaire des Hauts-de-Meuse dont l'altitude varie de 300 à 350 m (fig. 2). Le plateau domine la plaine argileuse de la Woëvre à l'est, dépression humide située entre 200 et $250 \mathrm{~m}$ d'altitude. La liaison entre le plateau et la plaine s'effectue par le talus des côtes de Meuse (Amat, 2015 ; Hupy, 2006 ; De Matos-Machado et al., 2016 ; De Matos-Machado, 2018). Le 28 juillet 1914, la Première Guerre mondiale fut déclarée. Néanmoins, il fallut attendre l'année 1916 et la bataille de Verdun pour observer de véritables changements dans les paysages meusiens. On compta entre 1 et 2 millions d'obus tombés lors des 21 et 22 février 1916, chiffre rehaussé à 60 millions pour les 300 jours de la bataille qui prit fin en décembre 1916 (Gascouin, 1920 ; Cochet, 2014 ; Prost et Krumeich, 2015). De la violence et de la durée des bombardements sur un territoire sensiblement équivalent à celui de la ville de Paris, a résulté une modification profonde et inédite du paysage. L'image d'une peau d'orange est aujourd'hui évoquée pour illustrer l'aspect chaotique du champ de bataille de Verdun (Hupy, 2006 ; Hupy et Schaetzl, 2006 ; De Matos-Machado et al., 2016). Les efforts de reboisement menés sur la décennie 1925-1935, associés à la politique du gouvernement d'après-guerre empêchant toute récupération des terres et restauration des villages, ont conduit dans les années 1940 à un paysage fortement boisé, ayant protégé le sol de 70 ans de pluie et d'érosion agricole (Steinbach et Husson, 2007 ; Amat, 2015). À l'inverse 
d'autres champs de bataille où les stigmates géomorphologiques se sont effacés avec le temps et les activités agricoles, celui de Verdun a conservé un relief quasiment inchangé depuis l'armistice. En ce sens, la forêt domaniale de Verdun forme un excellent site pour cartographier, analyser et classifier les polémoformes induites par la Grande Guerre, opérations rendues possibles depuis l'acquisition en 2013 de données LiDAR sur l'ensemble de la forêt.

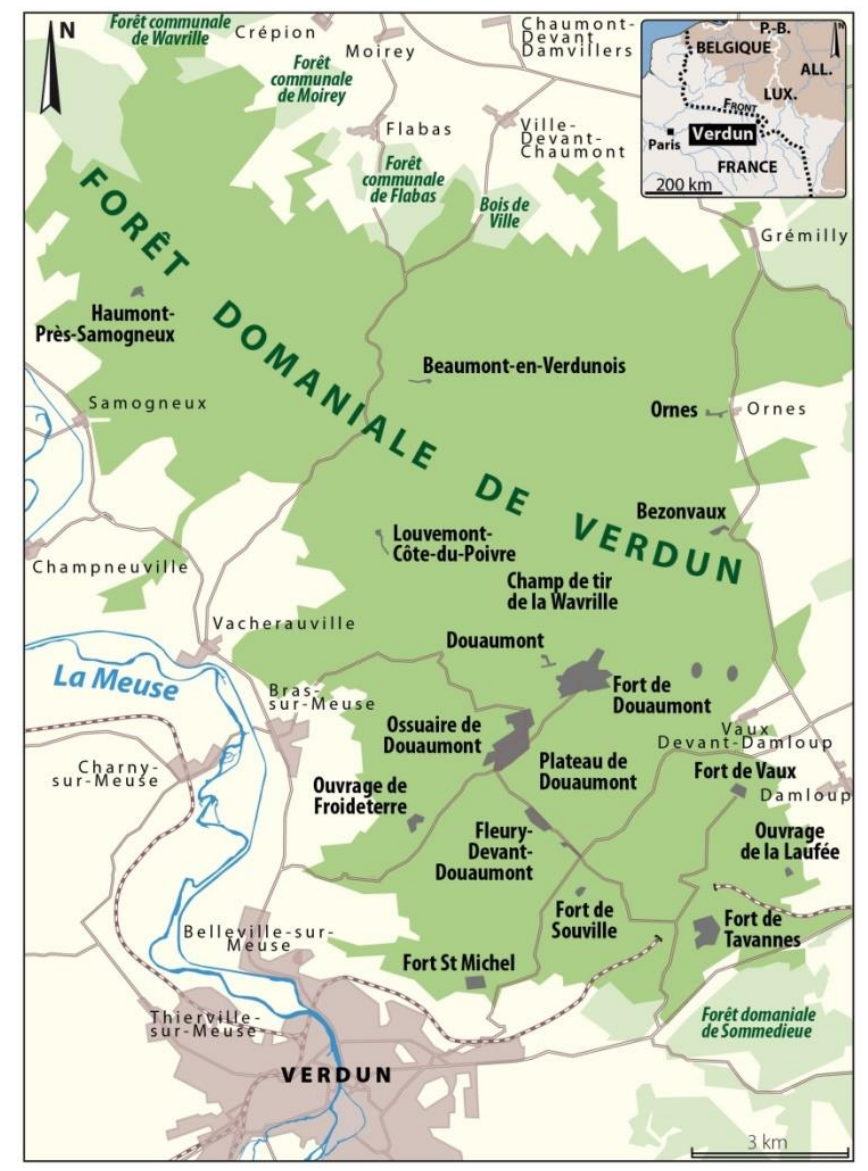

Figure 1. Localisation de la forêt domaniale de Verdun. D'après De Matos-Machado, 2018.

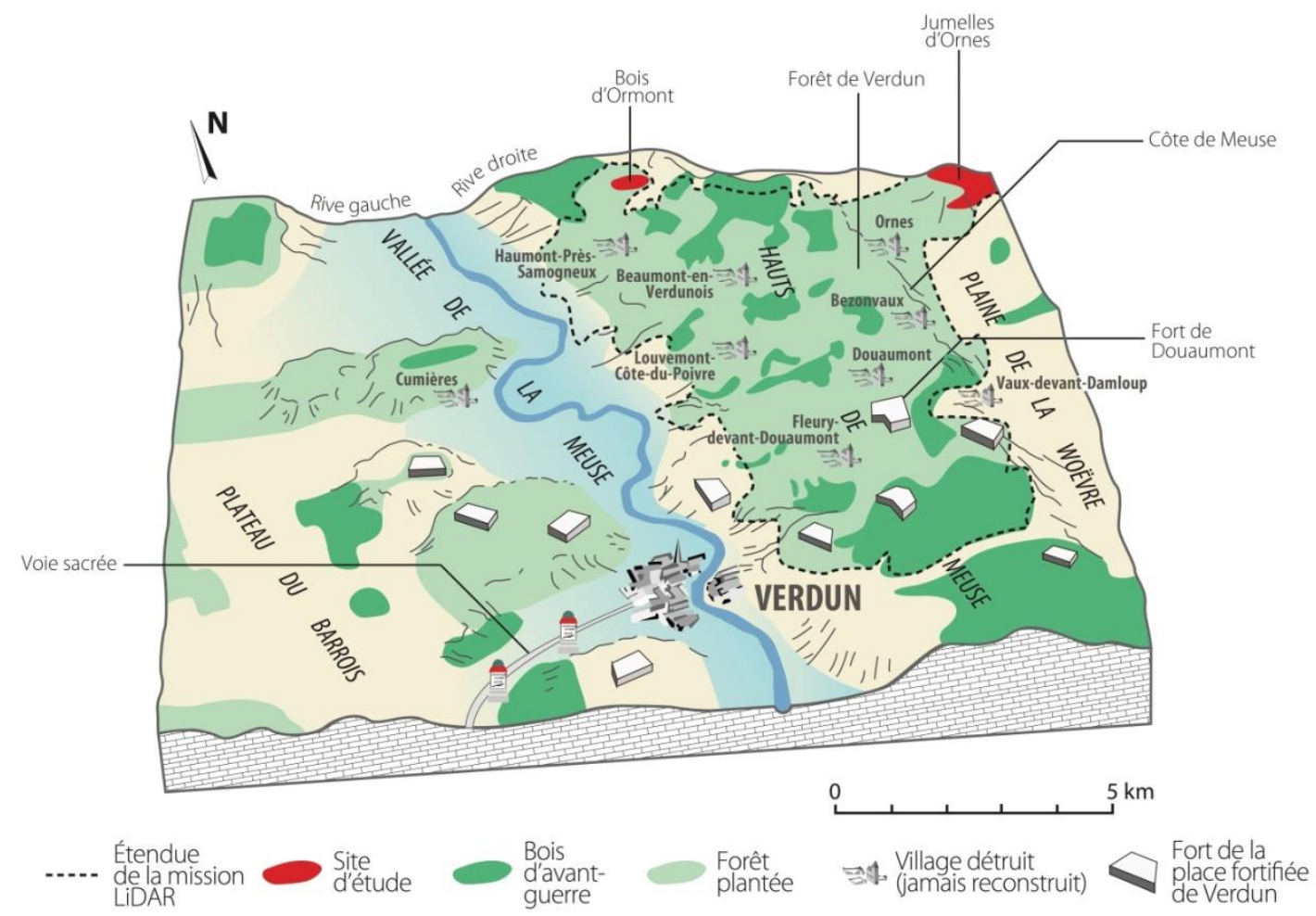

Figure 2. Localisation des sites échantillons. D'après De Matos-Machado et al., 2019. 


\section{Données et méthodes}

\section{Présentation des données LiDAR et des documents d'archives}

Le travail se fonde sur l'utilisation d'un jeu de données LiDAR obtenu par avion les 26 et 27 mars 2013. Cette mission aéroportée a été cofinancée par l'ONF (agence de Verdun), le Conseil départemental, le GIP Objectif Meuse, la région Lorraine, le Mémorial de Verdun et le SRA Grand-Est (site de Metz) dans le cadre du projet «Verdun 14-18, Forêt d'exception ». Elle a permis l'acquisition d'un nuage de points de $100 \mathrm{~km}^{2}$ recouvrant l'intégralité de la forêt domaniale de Verdun avec une densité moyenne de 44 points $/ \mathrm{m}^{2}$ au sommet de la canopée et 16 points $/ \mathrm{m}^{2}$ au niveau du sol. Dans l'objectif de visualiser la topographie du sol forestier, les points sol du nuage de points LiDAR ont été exploités pour générer un MNT de $20 \mathrm{~cm}$ de résolution. Cette étape a été accomplie à l'aide d'ArcGIS ${ }^{\circledR}$ Desktop 10.5 et la méthode des voisins naturels du jeu d'outils d'interpolation, jugée adaptée en raison de sa rapidité de calcul et de son efficacité sur des terrains plats (Bater et Coops, 2009). Par la suite, la méthode de débruitage développée par Sun et al. (2007) a été appliquée sur le MNT en utilisant l'outil Mesh Denoise de SAGA GIS ${ }^{\circledR}$, algorithme consistant à lisser les faibles rugosités de surface tout en conservant la forme générale du terrain. Cette étape a permis de gommer les structures indésirables du sous-bois (branches, troncs, aspérités naturelles du relief). Grâce à l'exploitation des documents d'archives, le MNT a pu être analysé pour faire apparaître les grandes métamorphoses géomorphologiques du champ de bataille de Verdun sur un siècle. Ces documents ont aussi permis de valider nos interprétations. Le MNT a ainsi été confronté à plus d'un millier de documents historiques récoltés dans huit centres d'archives allemands et français et dans le domaine privé (principalement des plans historiques et photographies aériennes).

\section{Calibration de l'algorithme sur deux sites échantillons}

Afin de rendre notre cartographie aussi fiable que possible, la méthode a été calibrée sur deux sites échantillons ayant bénéficié d'une série de prospections sur le terrain entre 2014 et 2016. Le premier site (290 ha) se trouve sur le plateau des Hauts-de-Meuse au nord-ouest de la forêt de Verdun et inclut le bois d'Ormont; le second site (420 ha) occupe les Jumelles d'Ornes, butte-témoin localisée à l'est de la forêt, en avant des côtes de Meuse et au nord du village reconstruit d'Ornes (fig. 2). Ces deux sites ont été sélectionnés pour la variété de polémoformes qu'ils abritent et pour leurs configurations géomorphologiques différentes, permettant de tester le comportement de l'algorithme sur différentes pentes. Les visites sur le terrain ont eu lieu durant les mois de mars et avril quand les conditions de visibilité du sous-bois étaient optimales. Elles ont été menées avec l'aide des agents forestiers de l'ONF et des archéologues du SRA en utilisant l'approche déployée en 2014 (De Matos-Machado et al., 2016). Pour chaque forme échantillonnée, les observations in situ ont consisté à identifier par analyse morphométrique (identification de creux ou de bosses, de pentes saillantes ou atténuées, d'un contour polygonal ou émoussé) leur origine et à séparer les structures aménagées par les soldats (tranchées, boyaux, abris, positions d'artillerie) de celles induites par les bombardements (cratères d'obus). Il a résulté de ces missions de terrain l'interprétation de 879 polémoformes. 


\section{Inventaire des formes}

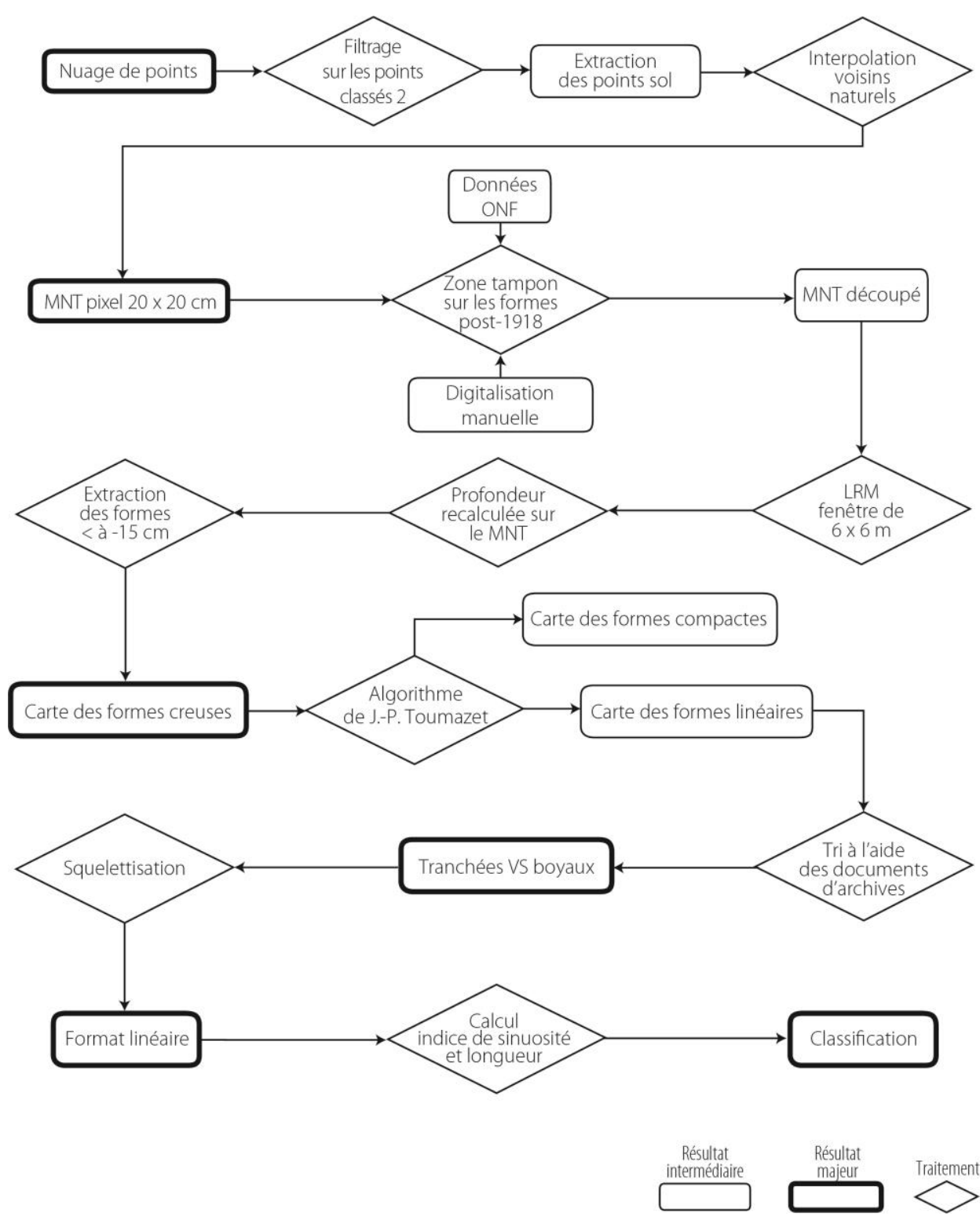

Figure 3. Chaîne de traitements décrivant la procédure d'inventaire des fossés.

Après avoir identifié les polémoformes sur le terrain, une méthode de cartographie a été développée afin d'extraire les formes visibles sur le MNT. L'algorithme, appliqué dans un premier temps sur les deux sites échantillons puis sur l'ensemble de la forêt de Verdun, se décline en cinq étapes distinctes (fig. 3) :

1. Les formes postérieures à la guerre (routes, cloisonnements et lisières marqués) ont été éliminées du MNT de manière à ne conserver que les reliefs susceptibles d'avoir été créés par les combats de 1914-1918. Les données vectorielles disponibles auprès de l'ONF ont servi à accomplir cette opération pour la majorité des formes. Les structures restantes ont été digitalisées manuellement en privilégiant l'usage du sky-view factor (Georges-Leroy et al., 2014). Par la suite, une zone tampon de taille variable selon la largeur des structures a été tracée autour de chaque forme et a ensuite servi de masque. Le résultat final consiste en un MNT filtré et dépourvu a priori de formes postérieures à 1918.

2. L'algorithme du local relief model (LRM; Hesse, 2010), disponible dans le logiciel Relief Visualization Toolbox (Kokalj et al., 2011 ; Zakšek et al., 2011 ; Relief Visualization Toolbox, 2015) a été appliqué au MNT en utilisant une fenêtre glissante de $6 \times 6 \mathrm{~m}$, aux dimensions adaptées à la taille des polémoformes. Cet algorithme consiste à extraire de la tendance topographique les petites formes de relief et à produire une image composée de valeurs négatives (concaves) et 
positives (convexes) en mètres (Challis et al., 2011; Kokalj et al., 2011; Štular et al., 2012; Georges-Leroy et al., 2014). Néanmoins, l'algorithme génère un lissage du relief parfois non négligeable sur les pentes fortes. Pour cette raison, les valeurs de profondeur ont été recalculées en utilisant le MNT original.

3. Un seuillage a été appliqué sur les résultats du LRM pour n'extraire que les formes potentiellement induites par la Première Guerre mondiale. D'après nos observations in situ, les polémoformes de la forêt de Verdun sont des formes excavées le plus souvent bordées de bourrelets périphériques convexes, ces derniers étant difficilement caractérisables en raison de leur forme émoussée. Pour cette raison, nous avons mis de côté les reliefs convexes pour ne retenir que les formes creuses aux valeurs métriques négatives. Après plusieurs essais, seuls les pixels situés en dessous de $-15 \mathrm{~cm}$ ont été inventoriés afin de supprimer de la cartographie les faibles aspérités de surface liées aux microreliefs, aux bois et végétaux du sous-bois.

4. Après avoir extrait les formes excavées situées en dessous du seuil de $-15 \mathrm{~cm}$, l'algorithme développé par l'un des auteurs (J.-P. Toumazet) a été utilisé pour séparer les formes compactes et les formes linéaires puisque celles-ci ont bénéficié de méthodes d'inventaire et d'analyses morphométriques différentes (traitements zonaux d'un côté, traitements linéaires de l'autre; cf. étape 5). Il s'agit d'une adaptation de l'algorithme développé dans Toumazet et al. (2017) et qui se fonde sur les mesures d'une emprise géométrique minimale (communément appelée bounding box), représentant le plus petit rectangle englobant une forme.

5. La dernière étape a consisté à préparer les deux couches précédemment obtenues en vue de leur analyse morphométrique. Pour les formes compactes (cratères d'obus, abris pour les personnes et le matériel, positions d'artillerie, etc.), une analyse multivariée emboîtée a été appliquée afin de discriminer les formes en fonction de leurs caractéristiques morphométriques. La démarche est explicitée en détail dans De Matos-Machado et al. (2019). Pour les formes linéaires (tranchées et boyaux de communication), sujet principal de cet article, un changement de représentation a été opéré, consistant à passer d'un format zonal à un format linéaire. Cette procédure, appelée squelettisation, a permis l'obtention d'une version simplifiée et linéique des polygones nommée squelette. Elle a été réalisée à l'aide de la boîte à outils en accès libre Polygon to Centerline Tool for ArcGIS développée par Dilts (2015a).

\section{Analyse morphométrique et distinction des tranchées et boyaux}

Pour évaluer l'étendue et la variabilité géométrique des fossés conservés au moment du relevé LiDAR (opération rendue possible après leur conversion en lignes), deux paramètres ont été calculés et stockés dans la table attributaire de chaque entité :

1. La longueur $(L)$ : celle-ci s'exprime en mètres et a été calculée dans $\operatorname{ArcGIS}^{\circledR}$ à l'aide de l'outil Calculer la géométrie de la table attributaire.

2. L'indice de sinuosité (Is) : couramment utilisé en hydrogéomorphologie pour caractériser le tracé des cours d'eau, cet indice défini par Brice (1964) est particulièrement pertinent pour décrire la morphologie des réseaux de fossés et peut être obtenu à l'aide de la boîte à outils Stream Gradient \& Sinuosity Toolbox d'ArcGIS ${ }^{\circledR}$ (Dilts, 2015b).

À l'inverse des formes compactes dont le nombre et la variété géométrique ont rendu pertinente leur classification automatique (De Matos-Machado et al., 2019), les formes linéaires n'ont pas bénéficié d'une telle analyse. Une classification sur la base des deux indices sus-cités $(L, I s)$ n'a pas permis d'établir de distinction entre tranchées et boyaux de communication en raison d'une trop grande proximité morphométrique. Cette catégorisation a été opérée par tri manuel en considérant les 
toponymes et l'orientation des fossés représentés sur les plans directeurs; la méthode d'inventaire proposée est donc semi-automatique.

\section{Analyse diachronique des formes}

Seule la période 1918-2013 a été étudiée dans cet article afin d'évaluer le taux d'effacement des formes sur un siècle. L'opération, réalisée sur les réseaux de tranchée et boyaux, a consisté à croiser le MNT de 2013 avec une vingtaine de plans directeurs et photographies aériennes représentatifs du champ de bataille au sortir de la guerre. Les fossés de 1918 représentés sur ces archives ont été entièrement digitalisés sur $\operatorname{ArcGIS}^{\circledR}$, permettant une quantification de leur longueur totale. En complément, la comparaison de la couche vectorielle ainsi créée avec la carte des formes de 2013 a permis le repérage des secteurs où ces fossés sont particulièrement fragmentés, voire ont totalement disparu. Ainsi, les archives nous donnent les moyens de localiser les secteurs les plus érodés où n'ont subsisté que quelques fragments de fossés.

\section{Résultats}

Longueur totale et distribution spatiale des fossés au moment du relevé LiDAR (2013)

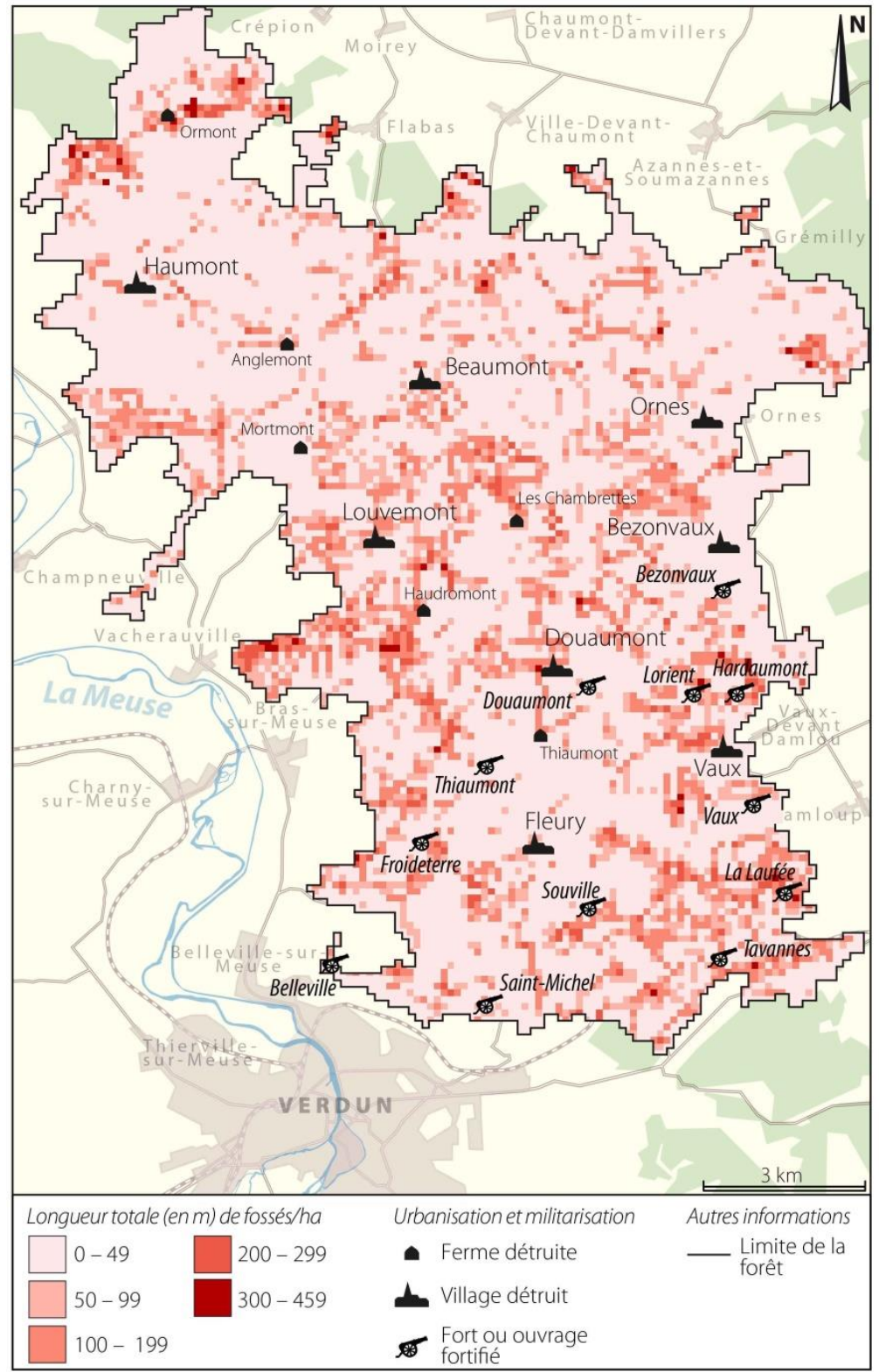

Figure 4. Carte de densité du réseau de fossés. Chaque pixel équivaut à 1 ha. D'après De Matos-Machado, 2018. 
La cartographie semi-automatisée des formes linéaires a donné lieu à l'inventaire de $424 \mathrm{~km}$ de fossés, soit une moyenne de $44 \mathrm{~m}$ de fossés/ha (fig. 4). Avec des valeurs de longueur $(L)$ comprises entre $0,24 \mathrm{~m}$ et $447 \mathrm{~m}(\bar{L}=24)$, le réseau apparaît particulièrement fragmenté et composé d'entités de petite taille. Le tracé de ces dernières est globalement sinueux avec un indice de sinuosité (Is) moyen de 1,12 pour un minimum et un maximum variant de 0 à 6,35 (tab. 1). L'exploitation des plans directeurs allemands et français différencie tranchées - fossés perpendiculaires au front - et boyaux parallèles au front -, dont le kilométrage est fixé à $145,63 \mathrm{~km}$ pour la première catégorie et 278,33 km pour la seconde. L'Is est en moyenne de 1,14 pour les tranchées contre 1,11 pour les boyaux. L'analyse approfondie de la distribution des valeurs de l'Is indique une prépondérance des sections rectilinéaires pour les boyaux ( $40 \%$ ), plus faible pour les tranchées (29\%), justifiant l'écart des moyennes de l'Is pour les deux catégories de fossés. La dispersion des fossés à l'échelle de la forêt est assez égale, mais laisse apparaître quelques agrégats. Cinq zones à forte densité s'individualisent. La première est la côte du Poivre, position fortement disputée au milieu de la bataille de Verdun où des densités comprises entre 50 et $400 \mathrm{~m} / \mathrm{ha}$ sont relevées. Un second amas allongé avec des valeurs aussi importantes s'étend du bois de Brabant au bois d'Ormont, arrière-front allemand transformé en forteresse entre 1917 et 1918. Un troisième site apparaît autour de l'ouvrage de la Laufée, position française où s'observent des maximas proches de $400 \mathrm{~m} / \mathrm{ha}$. Dans les secteurs de l'ouvrage d'Hardaumont et des Jumelles d'Ornes, les densités sont aussi importantes, entre 50 et $400 \mathrm{~m} / \mathrm{ha}$. Les fortes densités de tranchées indiquent des systèmes dédoublés (présence de deux ou trois lignes) ou de linéaires bien conservés (peu fragmentés) alors que les vides renseignent sur la localisation des zones de contact se matérialisant par un no man's land. Toutefois, ces zones à faible densité de tranchées ne doivent pas être interprétées systématiquement comme telles, d'autres facteurs doivent être pris en compte (destruction des tranchées par les bombardements, absence d'aménagement). Pour les boyaux de communication, les pixels aux valeurs élevées révèlent la présence de lieux bien connectés où les lignes se multiplient. De ce fait, les secteurs denses en boyaux de communication se révèlent être de bons indicateurs de présence d'abris, ces derniers nécessitant (pour la plupart) un raccordement au réseau.

\begin{tabular}{cccc}
\hline $\begin{array}{c}\text { Indice de } \\
\text { sinuosité }(I s)\end{array}$ & Type de tracé & $\begin{array}{c}\text { Part des } \\
\text { tranchées (\%) }\end{array}$ & $\begin{array}{c}\text { Part des boyaux } \\
(\%)\end{array}$ \\
\hline 0 & Boucle fermée & 0,03 & 0,02 \\
\hline 1 à 1,05 & Rectiligne; quasi rectiligne & 29,31 & 40,52 \\
\hline 1,06 à 1,10 & $\begin{array}{c}\text { Bastionné ou à redans espacés; tenaillés, à crémaillère ou en } \\
\text { vagues à faible longueur d'onde; rectilignes courbés (virage) }\end{array}$ & 22,53 & 23,69 \\
\hline \multirow{2}{*}{$\begin{array}{c}\text { Tenaillés, à crémaillère ou en vagues à longueur d'onde } \\
\text { modérée; courbés (virage) et tenaillés, à crémaillère ou en } \\
\text { vagues à longueur d'onde modérée }\end{array}$} & 25,17 & 21,48 \\
\hline 1,21 à 1,50 & $\begin{array}{c}\text { Bastionné ou à redans faiblement espacés; fossés tenaillés, à } \\
\text { crémaillère ou en vagues à longueur d'onde importante }\end{array}$ & 19,86 & 12,14 \\
\hline$>1,50$ & En traverses; courbés en traverses; boucle ouverte & 3,07 & 2,11 \\
\hline
\end{tabular}

Tableau 1. Interprétation de l'indice de sinuosité.

Sources : De Matos-Machado, 2018. 


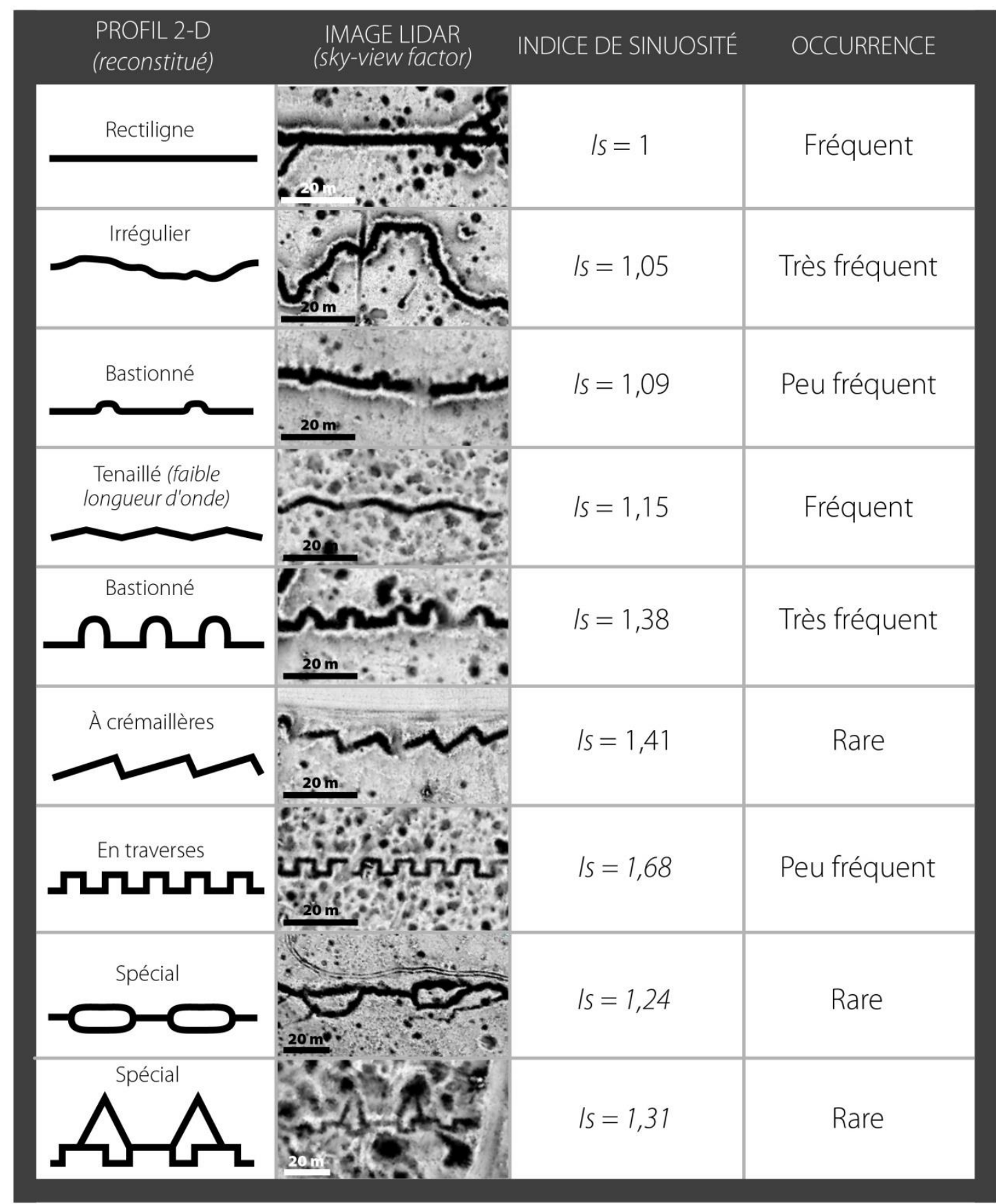

Figure 5. Extrait de la typologie des réseaux de fossés. Typologie complète dans De Matos-Machado, 2018.

Sur la base des résultats obtenus après calcul de l'Is et lecture de la carte d'inventaire, vingt-et-un types de tranchées et boyaux de communication ont été identifiés, aux tracés de formes et de sinuosités variées (fig. 5). Neuf types de tracés de tranchées et douze de boyaux de communication ont été relevés. Ces derniers occupent une part largement supérieure à celles des tranchées dans les fossés cartographiés (les deux tiers). Cette typologie, inédite dans le secteur, a été comparée à deux études de référence ayant étudié la variabilité morphologique des réseaux de fossés de la Première Guerre mondiale. Stichelbaut (2009) a identifié sur le front belge pas moins de dix tracés de tranchée et quinze tracés de boyaux de communication. Taborelli et al. (2017a) ont distingué trois tracés de tranchée et cinq tracés de boyaux. En faisant abstraction des différents moyens méthodologiques employés par ces études (photo-interprétation d'images aériennes, plans directeurs et/ou imagerie LiDAR), nous constatons par simple comptage des catégories que la variabilité morphométrique des tracés observés sur le champ de bataille de Verdun est forte. Alors que la surface prospectée est presque cinq fois plus petite que celle étudiée par Stichelbaut $\left(2009 ; 96,15 \mathrm{~km}^{2}\right.$ contre $\left.443,5 \mathrm{~km}^{2}\right)$, presque autant de catégories de fossés ont été relevées sur notre site d'étude. En complément, la plupart 
des sections cartographiées manquent de régularité dans leur tracé, constat inverse à ce que semblent indiquer les études sur les réseaux flamands et champenois. Ainsi, à Verdun, il n'est pas rare d'observer des tracés rectilignes ponctuellement sinueux au sein d'une même section.

\section{Taux d'effacement des fossés sur la période 1918-2013}

La carte des fossés de 1918 permet un regard objectif sur le nombre de polémoformes disparues au cours du dernier siècle et de proposer une cartographie du taux d'effacement des formes à l'unité parcellaire de la forêt (fig. 6a, 6b et 7). Ce maillage parcellaire a été privilégié pour la discrétisation puisque les aménagements forestiers, impliquant notamment des travaux d'entretien et de conservation, sont conduits à cette échelle. La carte produite pourra ainsi être directement exploitée par les gestionnaires forestiers. L'étude de la cartographie de 1918 révèle que le réseau de tranchées et boyaux atteignait $1032 \mathrm{~km}$ au sortir de la guerre. Les réseaux plus anciens, disparus sous l'effet de l'artillerie, ne sont pas comptabilisés et on peut imaginer que leur prise en compte augmenterait significativement ce chiffre. La comparaison des réseaux entre 1918 et 2013 révèle une déperdition de $608 \mathrm{~km}$ de fossés, soit une perte considérable de $59 \%$ du réseau initial.

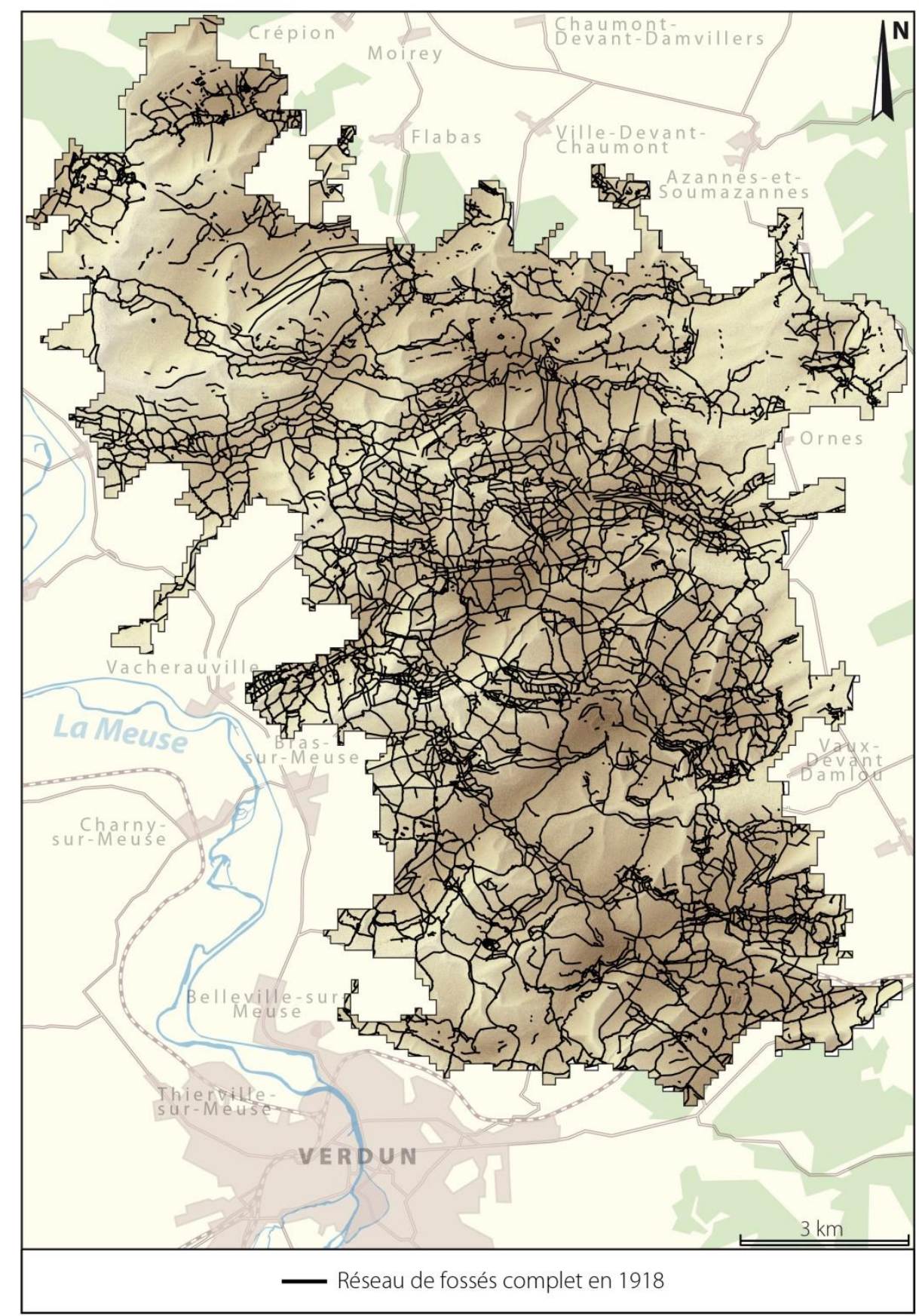

Figure 6a. Le réseau de fossés complet en 1918. D'après les photographies aériennes et plans directeurs allemands et français. 


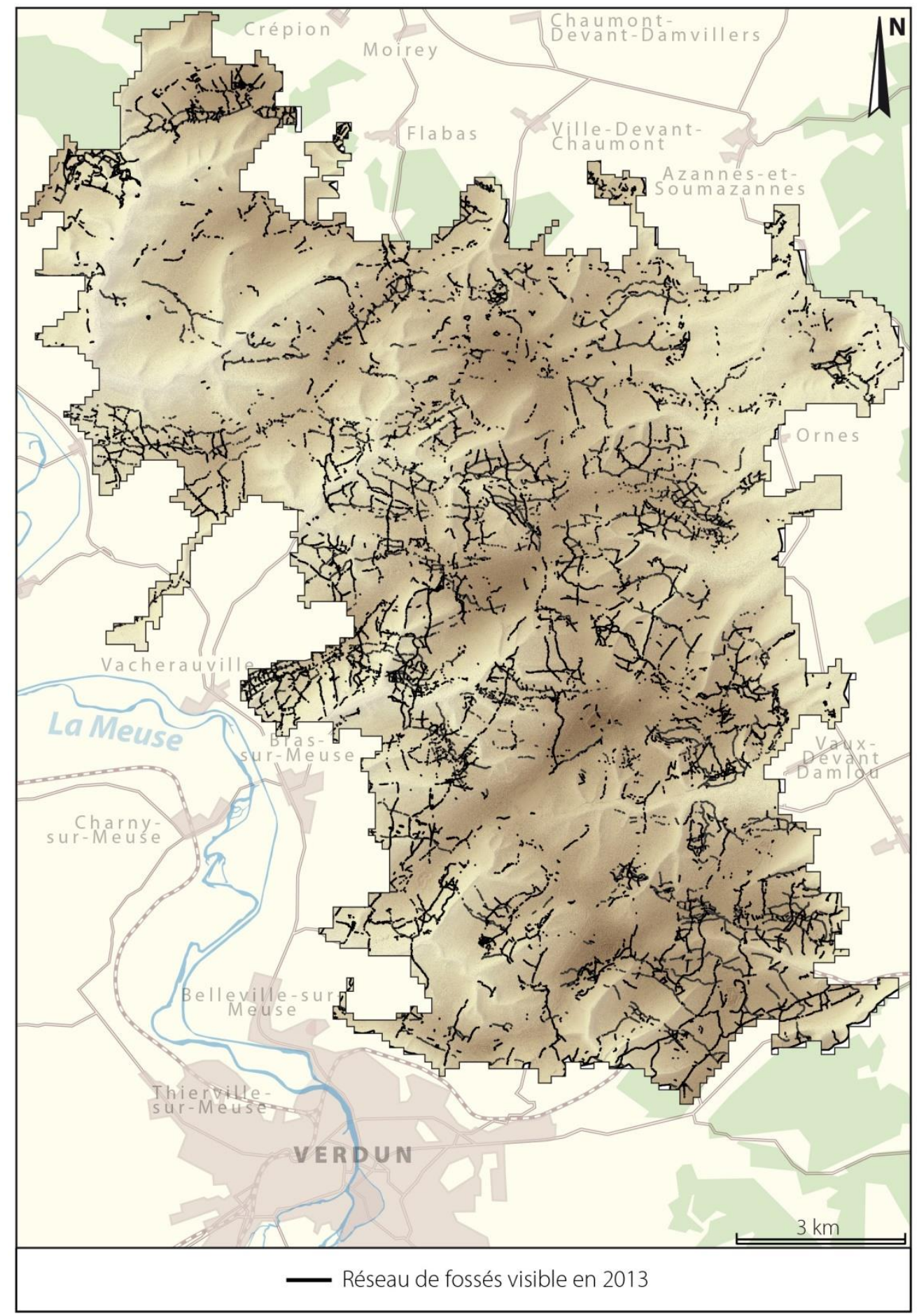

Figure 6b. Le réseau de fossés visible sur l'imagerie LiDAR de 2013. D’après les données LiDAR. 


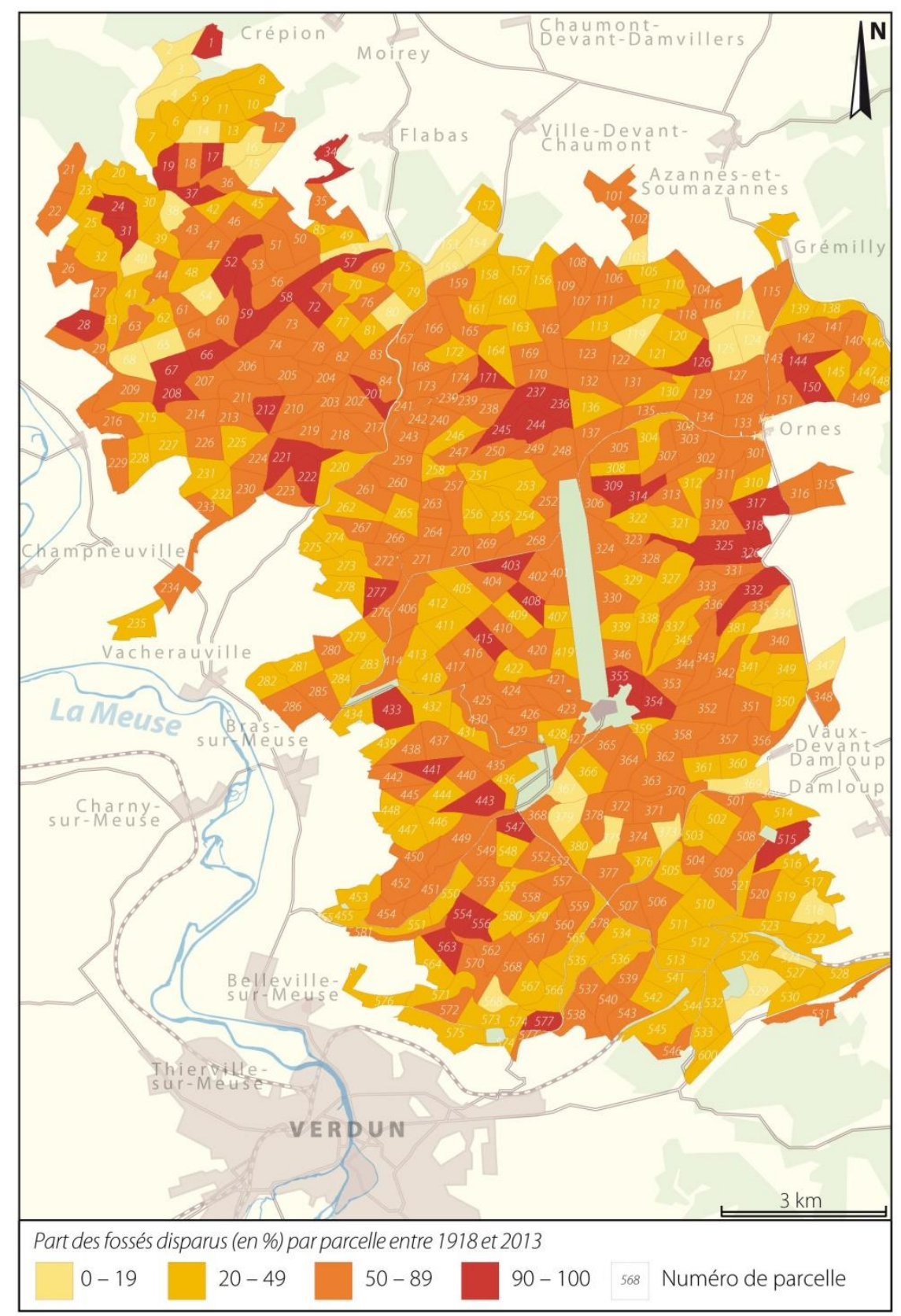

Figure 7. Taux d'effacement des fossés par parcelle. D’après De Matos-Machado, 2018.

\section{Discussion}

\section{Fiabilité de la cartographie semi-automatique des fossés}

Les observations in situ ont servi de moyen de calibration pour le développement d'une méthode d'inventaire semi-automatique des polémoformes. En ce qui concerne les fossés, l'ultime version de l'algorithme a permis d'inventorier sur les deux sites échantillons la quasi-totalité du réseau puisque sur les $16 \mathrm{~km}$ de fossés observés dans ces secteurs, seulement 440 m n'ont pas été repérés, soit un taux de détection de $97 \%$. Un certain nombre de raisons peuvent être invoquées pour justifier cette lacune dans la détection. Parmi les fossés non détectés, il existe des formes inondées. Dans son étude des mares de la forêt domaniale de Verdun, Printemps (2017) a montré l'inefficacité du LRM pour détecter les surfaces en eau puisque l'énergie émise par le LiDAR (proche infrarouge) a été partiellement, voire totalement absorbée par l'eau. D'autres formes n'ont pas été inventoriées, notamment quand leur profondeur se situait au-delà du seuil prédéfini (entre -15 et $0 \mathrm{~cm}$ ) lors de la phase d'extraction depuis le LRM. Étant donné l'importance du taux de détection, une digitalisation manuelle des fossés non détectés a été opérée à l'échelle de la forêt tout entière afin de compléter l'inventaire en utilisant l'indice sky-view factor, considéré comme particulièrement efficace pour identifier les formes linéaires 
creuses. Ainsi, $44 \mathrm{~km}$ de fossés supplémentaires ont été inventoriés de cette manière, permettant de répertorier a priori la totalité des formes linéaires observables par LiDAR.

\section{Variabilité morphométrique des fossés}

Différentes hypothèses peuvent être évoquées pour expliquer la grande richesse morphologique des fossés sur notre site d'étude (fig. 5). Une première concerne l'irrégularité géomorphologique et géologique du champ de bataille de Verdun, forçant les soldats à adapter constamment le tracé de leurs ouvrages en tenant compte du relief et du substrat (Schnitzler et Landolt, 2013). La variabilité morphologique des fossés dépend aussi de l'origine des soldats qui les ont aménagés, constat avancé par Stichelbaut (2009). Pour vérifier cela, six surfaces de taille identique (10 ha) et recouvertes de fossés ont été analysées, trois en camp allemand, trois en camp français (tab. 2). Les résultats de cette enquête montrent que la sinuosité des fossés est d'une façon générale plus importante côté allemand que côté français ( $I s=1,16$ contre 1,13 pour les tranchées ; $I s=1,12$ contre 1,11 pour les boyaux). Malgré la faible surface échantillonnée (60 ha) ne permettant pas de justifier d'écarts statistiquement significatifs - le test de Student indique une $p$-value de 0,14 pour les tranchées et 0,69 pour les boyaux avec un risque de $5 \%$-, des différences apparaissent sur le terrain entre les deux camps et pourraient refléter les deux doctrines de combats, la première privilégiant «le culte du retranchement» (esprit défensif allemand) alors que la deuxième misait sur «la rapidité du mouvement en avant» (esprit offensif français ; Normand, $1921: 5$ et 56). Une étude plus exhaustive menée sur un échantillon plus important de zones pourrait être envisagé pour préciser ces écarts.

\begin{tabular}{|c|c|c|c|c|c|}
\hline & Lieu & $\begin{array}{c}\text { Tranchées } \\
\text { (total en km) }\end{array}$ & $\begin{array}{l}\text { Tranchées } \\
\text { (Is moyen) }\end{array}$ & $\begin{array}{c}\text { Boyaux } \\
\text { (total en km) }\end{array}$ & $\begin{array}{c}\text { Boyaux } \\
\text { (Is moyen) }\end{array}$ \\
\hline \multirow{4}{*}{$\begin{array}{l}\text { Camp } \\
\text { allemand }\end{array}$} & Bois d'Ormont & 0,68 & 1,17 & 1,25 & 1,18 \\
\hline & Bois de Champneuville & 0,66 & 1,15 & 1,15 & 1,08 \\
\hline & Jumelles d'Ornes & 1,47 & 1,16 & 0,95 & 1,10 \\
\hline & Moyenne & - & 1,16 & - & 1,12 \\
\hline \multirow{4}{*}{$\begin{array}{c}\text { Camp } \\
\text { français }\end{array}$} & Côte du Poivre & 1,30 & 1,10 & 1,15 & 1,09 \\
\hline & Bois des Vignes & 0,17 & 1,15 & 1,01 & 1,11 \\
\hline & Tunnel de Tavannes & 0,97 & 1,14 & 0,73 & 1,12 \\
\hline & Moyenne & - & 1,13 & - & 1,11 \\
\hline
\end{tabular}

Tableau 2. Comparaison des réseaux de fossés allemands et français.

Sources : De Matos-Machado, 2018.

\section{Facteurs à l'origine de l'érosion des formes}

Le recouvrement du champ de bataille de Verdun par une forêt de 10000 ha, une décennie après la guerre, a eu pour effet de conserver une part importante des polémoformes (fig. 6b). Malgré cette initiative théoriquement conservatoire d'un point de vue archéologique et géomorphologique, la conduite d'un tel projet de boisement a nécessité des phases de nettoyage et de nivellement. Ces travaux se sont accompagnés de dégradations sur le relief, en particulier durant la première phase de boisement, engendrant localement la disparition totale de polémoformes (fig. 6a, 6b et 7). En complément des travaux sylvicoles, d'autres facteurs anthropiques sont à l'origine de dégradations des polémoformes. Les nombreux aménagements post-guerre (axes routiers, sites de mémoire) ont complètement fait disparaître la topographie passée. Au vu des milliers de personnes qui visitent chaque année la forêt domaniale, une partie des dégradations est imputable à l'activité et aux flux touristiques. Paradoxalement, les associations responsables de la patrimonialisation du site contribuent parfois à l'altération des modelés hérités de la guerre. Par exemple, les démarches de réaménagement du champ de bataille peuvent être destructrices lorsqu'elles ne sont pas réfléchies en amont et accompagnées par des professionnels de l'archéologie et de la gestion patrimoniale. Autre catégorie d'acteurs, les pilleurs sont les véritables destructeurs du patrimoine archéologique. En dépouillant la 
forêt de ses trésors archéologiques, ces voleurs non seulement saccagent les microreliefs (de par leur action de creusement), mais encore privent les archéologues et les historiens militaires d'informations indispensables à l'interprétation des vestiges de guerre. La part des processus naturels dans l'érosion des polémoformes ne doit pas non plus être négligée. Les travaux de Schwartz et al. (2003) et Hupy et Schaetzl (2008) ont notamment mis en évidence une tendance au remblaiement naturel des polémoformes de l'ordre de 20 à $65 \mathrm{~cm}$ par siècle.

\section{Conclusions}

L'un des objectifs du contrat de projet «Verdun 14-18, Forêt d'exception» pour la période 20142018 était d'améliorer les connaissances relatives aux patrimoines de la forêt domaniale de Verdun, territoire phare de la guerre de position de la Première Guerre mondiale. Dans ce cadre, notre travail a tenté de répondre aux attendus du projet en produisant des fonds documentaires solides et exploitables par les gestionnaires de la forêt. D'un point de vue quantitatif, le développement d'un algorithme de cartographie semi-automatisé a permis de découvrir $423 \mathrm{~km}$ de tranchées et boyaux de communication. Sur le plan morphométrique, vingt-et-un types de tracés, dont quelques géométries inédites absentes des travaux sous-mentionnés, ont été relevés sur l'ensemble du massif forestier. Ce catalogue de référence complète ainsi les quatre typologies de polémoformes existantes sur d'autres parties du front de l'Ouest (Stichelbaut, 2009; Devos et al., 2015; Taborelli et al., 2017a; Gheyle et al., 2018). Pour les archéologues et géomorphologues, un tel document sera un outil de reconnaissance des polémoformes de la Grande Guerre sur des sites voisins n'ayant pas encore bénéficié d'une telle approche. De plus, l'exploitation des documents d'archives a permis, au-delà de la simple interprétation des polémoformes, de caractériser l'état de conservation actuelle des fossés. Il en est ressorti l'identification de $608 \mathrm{~km}$ de fossés effacés, ce qui équivaut à une perte considérable de $59 \%$ du réseau initial (au sortir de la guerre). Ces résultats nous ont interrogés sur les processus responsables de l'atténuation de la topographie héritée de la Grande Guerre. Si la part de l'érosion naturelle peut être importante, l'érosion anthropique liée aux travaux sylvicoles et aux activités touristiques est le premier facteur de cette déperdition. Il s'avère que les parcelles nivelées et reconverties en essences résineuses, en particulier les boisements des années 1970-1980, sont les plus touchées par ce phénomène.

\section{Remerciements}

Les auteurs tiennent à remercier Gersende Gérard, Gérald Colin, René-Marc Pineau et Juliette Foltier, de l'Office national des forêts, pour la mise à disposition des connaissances et des moyens techniques et logistiques nécessaires à la réalisation de l'étude; Gilles Arnaud-Fassetta, François Bétard et Clélia Bilodeau, de l'université Paris-Diderot, et Jean-Paul Amat de l'université ParisSorbonne, pour leur expérience et leurs précieux conseils ; ainsi que Denis Jacquemot, Romain Perrier, Valentin Printemps et Théo Wohlschlegel, pour leur aide sur le terrain. Nous remercions aussi les trois relecteurs pour leurs remarques utiles et constructives qui ont permis d'améliorer le manuscrit.

\section{Bibliographie}

Amat, J.-P. 1987. «Guerre et milieux naturels : les forêts meurtries de l'Est de la France, 70 ans après Verdun », L'Espace géographique, 16, $3: 217-233$.

Amat, J.-P. 2001. «La forêt entre guerre et paix, 1870-1995 : étude de biogéographie historique sur l'arc meusien de l'Argonne à la Woëvre ». Thèse d'État, Lille, Université de Lille 1, 1285 p.

Amat, J.-P. 2015. Les forêts de la Grande Guerre : histoire, mémoire, patrimoine. Paris, Presses de l'université ParisSorbonne, $548 \mathrm{p}$.

Bater, C. W., Coops, N. C. 2009. "Evaluating error associated with lidar-derived DEM interpolation", Computers \& Geosciences, 35, 2 : 289-300.

Brenot, J., N. Saulière, C. Léty, P. Taborelli, B. Zélie, R. Blondeau, A. Devos, Y. Desfossés, 2017. "How much did the soldiers dig? A quantification of WW1 remains in Argonne, France", Geoarchaeology, 32, 5 : 534-548. 
Brice, J. C. 1964. "Channel patterns and terraces of the Loup River in Nebraska", United States Geological Society, Washington, Paper 422-D, 41 p.

Challis, K., P. Forlin, M. Kincey, 2011. "A generic toolkit for the visualization of archaeological features on airborne LiDAR elevation data", Archaeological Prospection, 18, 4 : 279-289.

Cochet, F. 2014. La Grande Guerre: fin d'un monde, début d'un siècle. 1914-1918. Paris, Perrin, ministère de la Défense, $517 \mathrm{p}$.

De Matos-Machado, R. 2018. «Paysages de guerre et LiDAR : de la caractérisation des polémoformes à la conservation des patrimoines naturel et culturel de la forêt domaniale de Verdun (Meuse, France) ». Thèse de doctorat, Paris, université Paris-Diderot, 589 p.

De Matos-Machado, R., J.-P. Amat, G. Arnaud-Fassetta, F. Bétard 2016. «Potentialités de l'outil LiDAR pour cartographier les vestiges de la Grande Guerre en milieu intra-forestier (bois des Caures, forêt domaniale de Verdun, Meuse) », ÉchoGéo, $38: 1-23$.

De Matos-Machado, R., J.-P. Toumazet, J.-C. Bergès, J.-P. Amat, G. Arnaud-Fassetta, F. Bétard, C. Bilodeau, J. P. Hupy, S. Jacquemot 2019. "War landform mapping and classification on the Verdun battlefield (France) using airborne LiDAR and multivariate analysis", Earth Surface Processes and Landforms, 44, 7 : 1430-1448.

Devos, A., P. Taborelli, M. Dodici, L. Chalumeau, J. Buridant, N. Bollot, A. Combaud, Y. Desfossés 2015. « Rôle des conditions géographiques sur l'organisation spatiale des réseaux de défense de la Grande Guerre. Application à la Champagne (région de Reims) », Physio-Géo, 9: 157-174.

Dilts, T. E. 2015a. Polygon to Centerline Tool for ArcGIS. Reno, University of Nevada. URL: http://www.arcgis.com/home/item.html?id=bc642731870740aabf48134f90aa6165

Dilts, T. E. 2015b. Stream gradient and sinuosity toolbox for ArcGIS 10.1. Reno, University of Nevada. URL : http://www.arcgis.com/home/item.html?id=c8eb4ce1384e45258ccba1b33c $\mathrm{d} 4 \mathrm{e} 3 \mathrm{cb}$

Gascouin, F. E. $\left(\mathrm{G}^{\mathrm{AL}}\right)$. 1920. L'évolution de l'artillerie pendant la guerre. Bibliothèque de philosophie scientifique, 287 p.

Georges-Leroy, M., L. Nuninger, R. Opitz 2014. «Lidar: technique de détection au service de l'archéologie», Techniques de l'ingénieur, in $215: 1-27$.

Gheyle, W., B. Stichelbaut, T. Saey, N. Note, H. Van Den Berghe, V. Van Eetvelde, M. Van Meirvenne, J. Bourgeois 2018. "Scratching the surface of war. Airborne laser scans of the Great War conflict landscape in Flanders (Belgium)", Applied Geography, 90 : 55-68.

Hesse, R. 2010. "LiDAR-derived Local Relief Models - A new tool for archaeological prospection", Archaeological prospection, 17, $2: 67-72$.

Hupy, J. P. 2006. "The long-term effects of explosive munitions on the WWI battlefield surface of Verdun, France", Scottish Geographical Journal, 122, 3 : 167-184.

Hupy, J. P., R. J. Schaetzl 2006. "Introducing "bombturbation", a singular type of soil disturbance and mixing", Soil Science, $171,11: 823-836$.

Hupy, J. P., R. J. Schaetzl 2008. "Soil development on the WWI battlefield of Verdun, France", Geoderma, 145, $1: 37$ 49.

Jacquemot, S. 2014. «Regard archéologique sur les villages martyrs de la Grande Guerre en Lorraine », Archéopages, $40: 140-147$.

Jacquemot, S., J.-P. Legendre (éds). 2011. Vestiges de guerres en Lorraine : le patrimoine des conflits mondiaux. Metz, Serpenoise, $232 \mathrm{p}$.

Kokalj, Ž., K. Zakšek, K. Oštir 2011. "Application of sky-view factor for the visualization of historic landscape features in lidar-derived relief models", Antiquity, 85, 327 : 263-273.

Landolt, M. 2018. «L'archéologie de la Grande Guerre : une nécessaire interdisciplinarité », Histoire et archéologie, 6 : $61-75$.

Magnini, L., C. Bettineschi, A. De Guio 2016. "Object-based shell craters classification from LiDAR-derived sky-view factor", Archaeological Prospection, 24, 3 : 211-223.

Normand $\left(\mathrm{C}^{\mathrm{EL}}\right)$. 1921. L'évolution de la fortification de campagne en France et en Allemagne. Paris, Berger-Levrault, $113 \mathrm{p}$. 
Note, N., W. Gheyle, H. Van Den Berghe, T. Saey, J. Bourgeois, V. Van Eetvelde, , M. Van Meirvenne, B. Stichelbaut 2018. "A new evaluation approach of World War One's devastated front zone: A shell hole density map based on historical aerial photographs and validated by electromagnetic induction field measurements to link the metal shrapnel phenomenon", Geoderma, 310, 257-269.

Printemps, V. 2017. «Utilisation de l'outil LiDAR pour la détection des mares de la forêt domaniale de Verdun (Meuse) ». Mémoire de Master 1, Université Paris-Diderot, Paris, 96 p.

Prost, A., G. Krumeich 2015. Verdun 1916 : une histoire franco-allemande de la bataille. Paris, Tallandier, 318 p.

Relief visualization toolbox (RVT) 2015. URL : iaps.zrc-sazu.si/node/67731\#v

Schnitzler, B., M. Landolt (éds) 2013. À l'est, du nouveau! Archéologie de la Grande Guerre en Alsace et en Lorraine. Strasbourg, Éditions des Musées de la Ville de Strasbourg, 368 p.

Schwartz, C., L. Florentin, J.-L. Morel 2003. «Impact d'activités militaires de la Première Guerre mondiale sur la qualité des sols». Programme Life «Héritage bioculturel des forêts», ENSAIA-INPL, INRA, Laboratoire Sols et Environnement, Vandœuvre-lès-Nancy, 26 p., non publié

Steinbach, F., J. Husson 2007. «Palimpsestes et héritages des polémopaysages dans les massifs du Saillant de SaintMihiel », in : J.-L. Dupouey, E. Dambrine, C. Dardignac \& M. Georges-Leroy (éds), La mémoire des forêts. Nancy, Actes du colloque «Forêt, archéologie et environnement », ONF, INRA, DRAC Lorraine : 285-294.

Stichelbaut, B. 2009. "World War One aerial photography: An archaeological perspective". Thèse de doctorat, Universiteit Gent, Gand, 434 p.

Stichelbaut, B., D. Cowley (éds). 2016. Conflict landscapes and archaeology from above. Farnham, Ashgate publishing, $301 \mathrm{p}$.

Stichelbaut, B., W. Gheyle, T. Saey, V. Van Eetvelde, M. Van Meirvenne, N. Note, H. Van Den Berghe, J. Bourgeois 2016. "The First World War from above and below. Historical aerial photographs and mine craters in the Ypres Salient", Applied Geography, 66, 64-72.

Stichelbaut, B., N. Note, T. Saey, D. Hanssens, H. Van Den Berghe, J. Bourgeois, M. Van Meirvenne, V. Van Eetvelde, W. Gheyle 2017. "Non-invasive research of tunneling heritage in the Ypres Salient (1914-1918) - research of the Tor Top tunnel system", Journal of Cultural Heritage, 26, 109-117.

Štular, B., Ž. Kokalj, K. Oštir, L. Nuninger 2012. "Visualization of lidar-derived models for detection of archaeological features", Journal of archaeological science, 39, $11: 3354-3360$.

Sun, X., P. L. Rosin, R. R. Martin, F. C. Langbein 2007. "Fast and effective feature-preserving mesh denoising". IEEE Transactions on Visualisation and Computer Graphics, 13, 5 : 925-938.

P. Taborelli, A. Devos, S. Laratte, J. Brenot, N. Bollot, B. Cances, Y. Desfossés 2017a. «Apport des plans directeurs et de l'outil LiDAR aéroporté pour la caractérisation des impacts morphologiques de la Grande Guerre : exemple de la cote 108 (Berry-au-Bac, France)», Géomorphologie : relief, processus, environnement, 23, 2 : 155-169.

Taborelli, P., A. Devos, Y. Desfossés, J. Brenot, S. Laratte 2017b. «Typologie et organisation spatiale des «polémoformes » de la Grande Guerre révélées par l'outil LiDAR et les Plans directeurs. Application à la Champagne et à l'Argonne », Revue de géographie historique, 10-11.

Toumazet, J.-P., F. Vautier, E. Roussel, B. Dousteyssier 2017. "Automatic detection of complex archaeological grazing structures using airborne laser scanning data", Journal of Archaeological Science: Reports, 12 : 569-579.

Zakšek, K., K. Oštir, Ž. Kokalj 2011. "Sky-view factor as a relief visualization technique". Remote sensing, 12, 3 : 398 415. 